\title{
Ionizing Radiation and Hot Carrier Effects in SiC MOS Devices
}

\author{
E. A. de Vasconcelos, E. F. da Silva Jr., \\ Departamento de Física, Universidade Federal de Pernambuco, \\ Cidade Universitária, 50570-901 Recife, Pernambuco, Brazil \\ T. Katsube, and S. Yoshida \\ Graduate School of Science and Engineering, Saitama University \\ 255 Shimo-Okubo, Urawa-shi, Saitama-ken, 338-8570, Japan.
}

Received on 23 April, 2001

\begin{abstract}
We performed comparative studies of radiation-induced interface trap generation at n- $6 \mathrm{H}-\mathrm{SiC} / \mathrm{SiO}_{2}$ and $\mathrm{Si} / \mathrm{SiO}_{2}$ interfaces for high doses of low energy x-rays in the range 0 to $200 \mathrm{Mrad}(\mathrm{Si})$. We found that radiation-induced interface trap generation at the $\mathrm{n}-6 \mathrm{H}-\mathrm{SiC} / \mathrm{SiO}_{2}$ interface is much lower than at the $\mathrm{Si} / \mathrm{SiO}_{2}$ interface. The experiments provide evidence of interface trap generation near the $\mathrm{SiC}$ conduction band associated with oxide traps.
\end{abstract}

A microscopic model of radiation-induced defects in $\mathrm{SiC}$ MOS devices is still missing and further investigation is necessary [1]. In this work, we performed comparative investigations of ionizing radiation and hot carrier effects in $\mathrm{SiC}$ and $\mathrm{Si}$ MOS devices. We will report on experiments involving interface and oxide trap generation in the oxide and briefly discuss the mechanisms responsible for these effects.

Si MOS capacitors were fabricated using n-type 2 in., (100) silicon wafers of resistivity $1 \Omega$-cm. The wafers were cleaned by a modified RCA process as described elsewhere, yielding devices with radiation hardness improved by up to one order of magnitude [2]. Thermal oxides were grown in dry $\mathrm{O}_{2}$ at $1000^{\circ} \mathrm{C}$, followed by an in situ dry $\mathrm{N}_{2}$ annealing for 30 minutes. Aluminum films approximately $200 \mathrm{~nm}$ thick were thermally evaporated to form gate electrodes. After backside metallization, the wafers were annealed in forming gas at $400^{\circ} \mathrm{C}$ for 30 minutes. This is an optimized fabrication process yielding devices with improved radiation hardness. On the other hand, the processing of the SiC MOS devices was not optimized and the devices are not expected to have the best radiation hardness possible. $\mathrm{SiC}$ MOS capacitors were fabricated on a $3.5^{\circ}$ off-angled n-type $6 \mathrm{H}$-SiC wafer ( $\mathrm{Si}$ face) with a 5 -microns-thick epitaxial layer. The nominal doping density of the epilayer is $6 \times 10^{15} \mathrm{~cm}^{-3}$. The wafers were ultrasonically cleaned in successive, five-min steps in methanol, acetone and $10 \% \mathrm{HF}$ with a two-min rinse in deionized water between steps. The oxidation was performed in dry $\mathrm{O}_{2}$ at $1000^{\circ} \mathrm{C}$ for 13 hours, followed by an in situ dry $\mathrm{N}_{2}$ annealing for 30 minutes at the growth temperature. Aluminum was evaporated to form gate electrodes with a diameter of $1 \mathrm{~mm}$ and a large area back contact. The post-metallization annealing was done at $400^{\circ} \mathrm{C}$ in a mixture of $75 \%$ Ar- $25 \% \mathrm{H}_{2}$ during 30 minutes. Irradiation source was a $\mathrm{x}$-ray beam generated from a $\mathrm{Cu}$ target bombarded by $40 \mathrm{keV}$ electrons $(20 \mathrm{~mA})$ and the dose rate was $6 \mathrm{Mrad}(\mathrm{Si}) / \mathrm{h}$. Most of the absorbed dose results from the radiation near $10 \mathrm{keV}$. The x-ray dose was previously evaluated by means of dosimeters, which consist in Si photodiodes with an aluminum gate of specified thickness (200 nm). Irradiated samples always have aluminum gates with same electrode thickness $(200 \mathrm{~nm})$, so that the effective x-ray dose in the oxide is known. The capacitors were in open-circuit condition during irradiation. Carrier injection was performed by means of a HP 4155A Semiconductor Parameter Analyzer.

Exposure to ionizing radiation or hot carrier injection cause increasing degradation of Si- and SiC- based devices due to the generation of interface trap and oxide defects, as depicted in figures 1 and 2. Fig. 1 shows that after Fowler-Nordheim injection of electrons into the oxide, the current density increases in the direct and Fowler-Nordheim tunneling regimes. This increment in current is mostly associated with the generation of oxide traps, which assist the tunneling process. Oxide trap generation leads to oxide charging, soft and hard breakdown of the oxide. Oxide thickness and gate area 


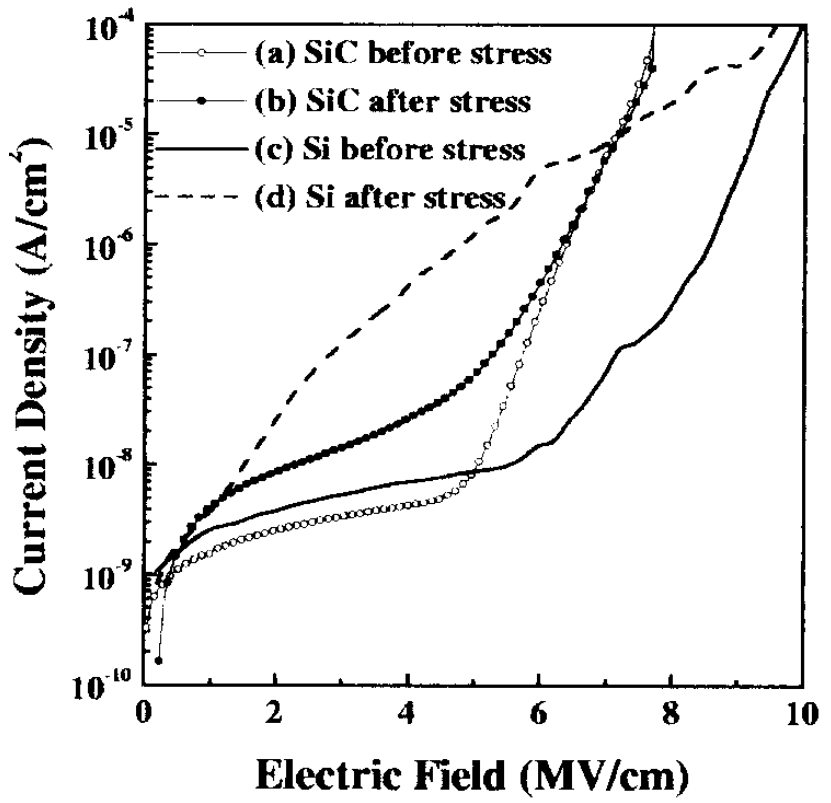

Figure 1. Current characteristics of $\mathrm{SiC}$ and Si MOS capacitors after repeated positive voltage ramp stressing (seven cycles, current limit set to $1 \mu \mathrm{A}$ ).

are respectively: $50 \mathrm{~nm}$ and $3.02 \times 10^{-3} \mathrm{~cm}^{2}$ (curves (a) and (b)) and $10 \mathrm{~nm}$ and $3.6 \times 10^{-3} \mathrm{~cm}^{2}$ (curves (c) and (d)). Fig. 2 shows room temperature capacitancevoltage characteristics of $\mathrm{Si}$ and $\mathrm{SiC}$ MOS capacitors irradiated with similar x-ray doses. Oxide thickness of the $\mathrm{n}-6 \mathrm{H}-\mathrm{SiC}$ MOS capacitor (curves (a) and (b)) is 85 $\mathrm{nm}$, gate area is $7.85 \times 10^{-3} \mathrm{~cm}^{2}$. Oxide thickness of the Si MOS capacitor (curves (c) and (d)) is $50 \mathrm{~nm}$, gate area is $1.22 \times 10^{-2} \mathrm{~cm}^{2}$. In Fig. 1, one can see a reduced leakage current generation in the $\mathrm{SiC}$ MOS device and analyzing the high-frequency capacitance-voltage (CV) curves in Fig. 2, it is clear that the negative voltage shift, reflecting the generation of positive oxide charge and the stretch-out of the curves, reflecting the generation of interface states, are much reduced in the $\mathrm{SiC}$ MOS capacitor. This striking difference in behavior after exposure to ionizing radiation was the main focus of this investigation. In order to estimate the total interface trap density, we used both the stretch-out technique and the photo-capacitance-voltage method (also known as the Jenq technique) [3], by illuminating the capacitors with intense white light from fiber optic illuminators for 5 to 10 minutes. We used roomtemperature quasistatic $\mathrm{CV}$ curves to check the quality of the oxide and to get a qualitative picture of the interface-trap generation. At room temperature, due to the large band gap of $\mathrm{SiC}$, minority carrier generation is very low and $\mathrm{SiC}$ behaves in similar way as $\mathrm{Si}$ at lower temperatures, so that the energy range probed by the high-low technique is reduced to energies very close to $\mathrm{SiC}$ conduction band, or equivalently, very close to accumulation. This region of the spectrum has been ascribed to oxide traps [1].

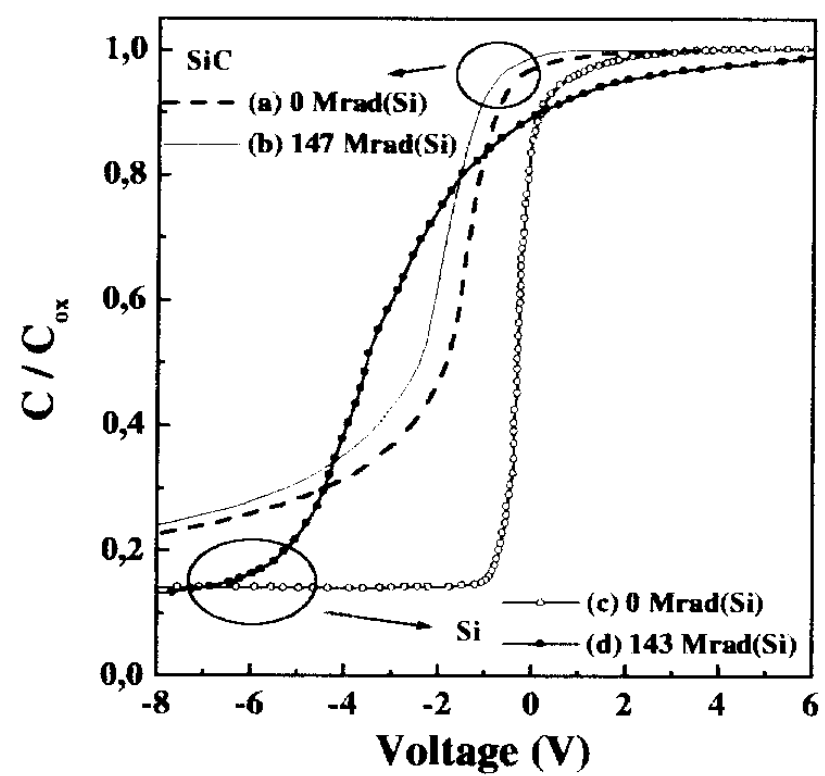

Figure 2. Capacitance-voltage characteristics of a Si MOS and a $\mathrm{SiC}$ MOS capacitor irradiated with similar x-ray doses.

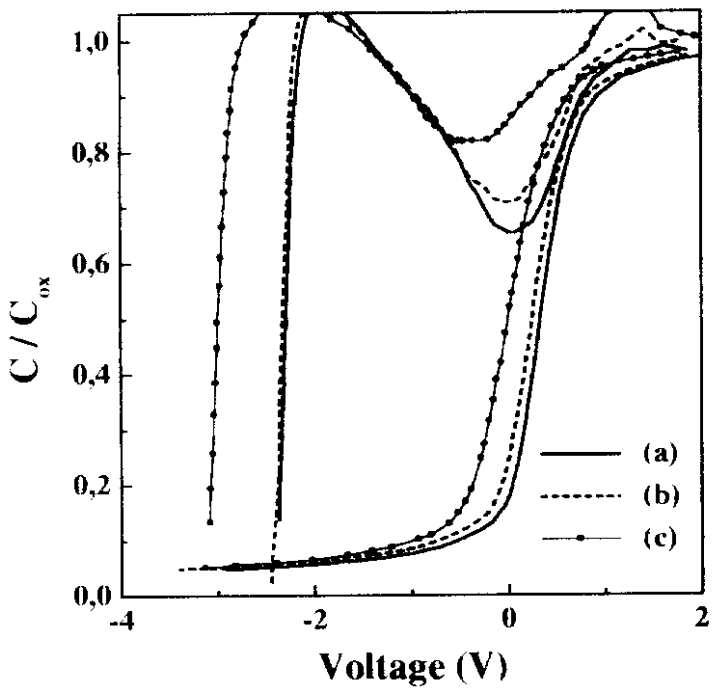

Figure 3. Capacitance-voltage curves of a $6 \mathrm{H}-\mathrm{SiC}$ MOS capacitor exposed to different $\mathrm{x}$-ray doses. Oxide thickness is $9 \mathrm{~nm}$ and gate area is $7.85 \times 10^{-3} \mathrm{~cm}^{2}$. Doses in $\operatorname{Mrad}(\mathrm{Si})$ are: (a) 0, (b) 14, (c) 110 .

Fig. 3 shows high frequency and quasistatic $\mathrm{CV}$ curves of a $\mathrm{SiC}$ MOS capacitor exposed to different $\mathrm{x}$-rays doses. As in Fig. 2, the negative voltage shift of the high-frequency $\mathrm{CV}$ curves, reflecting the generation of positive oxide charge is low. Most of the variation in the quasistatic curves appears near the accumulation region. This variation is probably due to the generation of interface states in the upper range of the $\mathrm{SiC}$ band gap, since the energy range probed by the technique is reduced to energies very close to $\mathrm{SiC}$ conduction band. Therefore, the variation observed in the quasistatic curves implies defect generation near the conduction band. States deeper into the bandgap are not detected and nothing can be said about them. 
Current peaks and misalignment are also observed. Current peaks near flatband are observed in devices with oxides heavily contaminated with mobile ions [5]. At elevated temperatures, when the oxide field reverses sign, the ions move and a peak in the displacement current appears. At room temperature, movement of the ions is reduced and we are led to believe that the current peaks are possibly related to oxide-trapped charges generated by irradiation and exchanging charge with the substrate, the so-called border traps or, equivalently, switching oxide traps [6]. Further investigation is needed to separate unambiguously the effect of the oxide traps from the effect of the mobile ions.

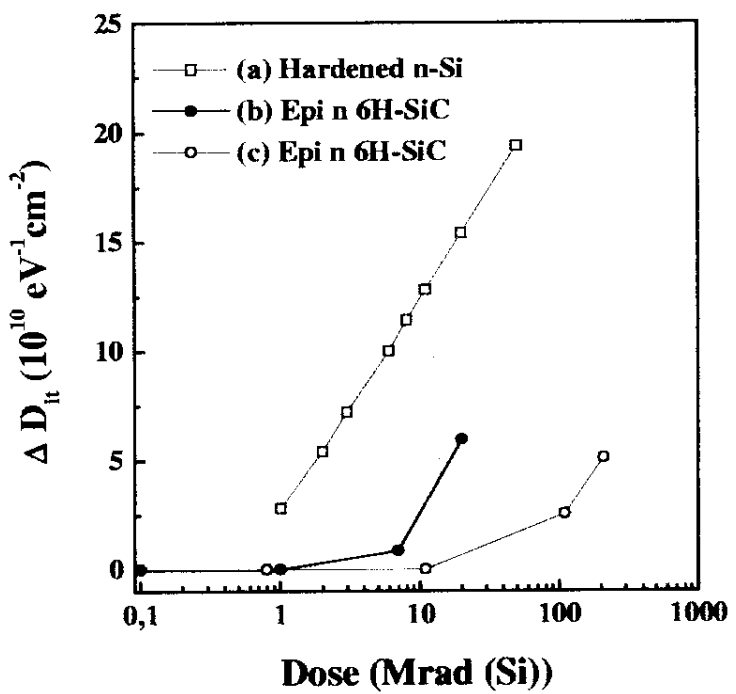

Figure 4. Interface trap generation in n-type $6 \mathrm{H}-\mathrm{SiC}$ and $\mathrm{Si}$ MOS capacitors. Curve(a) is a previously reported result obtained in n-type Si under similar irradiation conditions, as discussed in the text. Oxide thickness is $23 \mathrm{~nm}$ (curve (a)), $85 \mathrm{~nm}$ (curve (b)) and $9 \mathrm{~nm}$ (curve (c)).

Fig. 4 shows interface trap generation $\left(\Delta \mathrm{D}_{i t}\right)$ as a function of dose compared with a previously reported result obtained in n-type $\mathrm{Si}$ under similar irradiation conditions (curve(a)). Curve (a) is the response of a commercial radiation-hard device (23 $\mathrm{nm}$ gate oxide) exposed to $10 \mathrm{keV}$ x-rays at a dose rate of $18 \mathrm{Mrad}$ $(\mathrm{Si}) / \mathrm{h}$ with gate voltage equal to zero [4]. It is known, for $\mathrm{Si}$ devices, that the number of radiation-induced interface traps increases as the oxide thickness increases. Such a dependence on oxide thickness is also observed for the SiC devices, as shown in Fig. 4. However, it is clear that the $\mathrm{SiC}$ MOS capacitor has lower interface trap buildup, despite its oxide being almost four times thicker $(85 \mathrm{~nm})$ than the oxide of the commercial hardened $(23 \mathrm{~nm})$ Si device. The $\mathrm{SiC}$ MOS is radiationhard even without adopting radiation-hardening proce- dures. These results are consistent with previous reports, performed using lower doses of radiation, showing promising radiation hardness for $\mathrm{SiC}$ devices [7]. Radiation-induced holes and hydrogen ions migrating towards the $\mathrm{Si} / \mathrm{SiO}_{2}$ interface play a very important role in the formation of interface traps. It is known that a relatively thick $(20 \mathrm{~nm})$ negative charge layer has been observed in 6H-SIC MOS capacitors [7]. So, one possibility to explain improved radiation hardness in $\mathrm{SiC}$ MOS devices is to admit that a mechanism of annihilation or charge compensation occurs near or at the $\mathrm{SiC} / \mathrm{SiO}_{2}$ interface, therefore preventing the formation of interface traps by holes and hydrogen ions. The oxide traps generated by these compensation/annihilation reactions near the interface could act as oxide-trapped charges that exchange charge with the substrate, possibly causing peaks in the quasistatic CV curves.

In summary,comparative x-ray irradiation of epitaxial 6H-SiC MOS capacitors and Si MOS capacitors showed that interface trap generation rate is much reduced in $\mathrm{SiC}$ MOS devices. Evidence of interface trap generation near the $\mathrm{SiC}$ conduction band associated with switching oxide traps could be observed. The radiation response of $\mathrm{SiC}$ devices, under x-ray exposure, indicated a much higher radiation immunity than in $\mathrm{Si}$ devices, despite the fact that the $\mathrm{SiC}$ devices were not specially processed to be radiation hard.

\section{Acknowledgments}

The authors are thankful to the financial support received during the development of this work from MONBUSHO \# 08455160 (Japan), CNPq, PADCT/FINEP under contract \# 77.97.1120.00, and CTPETRO/FINEP under contract \# 65.00.02.80.00.

\section{References}

[1] V. V. Afanasev, Microeletr. Engin. 48, 241 (1999).

[2] E.F. da Silva Jr.,Y. Nishioka, and T. P. Ma, IEEE Trans. Nucl. Sci., NS-34, 1190 (1987).

[3] J. A. Cooper Jr, Phys. Status Solidi A 162,305 (1997).

[4] M. P. Baze, R. E. Plaag, and A. H. Johnston, IEEE Trans. Nucl. Sci, NS-36, 1858(1989).

[5] M. Kuhn and D. J. Silversmith, J. Electrochem. Soc. 118, 966 (1971).

[6] T. R. Oldham, Ionizing Radiation Effects in MOS Oxides, (World Scientific, Singapore, 1999) Chapter 2.

[7] M. Yoshikawa, K. Saitoh, T. Ohshima, H. Itoh, I. Nashiyama, S. Yoshida, H. Okumura, Y. Takahashi. and K. Ohnishi, J. Appl. Phys. 80, 282 (1996). 